\title{
PRÁCTICAS DE ALIMENTACIÓN DE LA POBLACIÓN INDÍGENA DEL DEPARTAMENTO DE CHOCÓ
}

\author{
${ }^{1}$ Andrea Hernández Quirama, ${ }^{2}$ Claudia Milena Velasco Rangel, ${ }^{3}$ María del Pilar Oviedo Cáceres, \\ ${ }^{4}$ Blanca Patricia Mantilla Uribe, ${ }^{5}$ Nieves Zoraida Flórez García. \\ ${ }^{1}$ Trabajadora Social. Ph.D. en Investigaciones Feministas y Género. Instituto PROINAPSA. Universidad Industrial de Santander. \\ ${ }^{2}$ Enfermera, maestrante en Pedagogía. Instituto PROINAPSA. Universidad Industrial de Santander. \\ ${ }^{3}$ Optómetra. Mg. en Salud Pública. Mg. en Educación para la Salud. Instituto PROINAPSA. Universidad Industrial de Santander. \\ ${ }^{4}$ Enfermera, Mg. en Pedagogía. Instituto PROINAPSA, Centro Colaborador de la OMS/OPS en Promoción de la Salud y \\ Salud Sexual Reproductiva. Universidad Industrial de Santander, Bucaramanga \\ ${ }^{5}$ Enfermera, Esp. en Gerencia de Empresas. Instituto PROINAPSA. Universidad Industrial de Santander.
}

Autor responsable de correspondencia: Blanca Patricia Mantilla Uribe.

Correo electrónico: bpmantil@uis.edu.co

\section{RESUMEN}

Objetivo: identificar las prácticas de conservación, manipulación, preparación y consumo de los alimentos de las comunidades indígenas del Departamento de Chocó.

Materiales y métodos: se realizó un estudio cualitativo descriptivo de tipo exploratorio. Por una parte, se utilizó la revisión documental de la política de seguridad alimentaria del país y, por otra, las indagaciones con las comunidades indígenas relacionadas con su percepción de las prácticas abordadas. Se realizaron conversatorios en total con 185 personas representantes de autoridades étnicoterritoriales, líderes indígenas en salud, hombres, mujeres, niñas y niños indígenas.

Resultados: las comunidades presentan falencias en cuanto a disponibilidad, acceso, consumo, utilización y calidad de los alimentos, los cuales son críticos para la seguridad alimentaria de las familias de las comunidades indígenas del departamento.

Conclusiones: la situación que viven las comunidades indígenas del Chocó es de inseguridad alimentaria, además, tienen dificultades de acceso a agua potable, saneamiento básico, entre otros, lo que los ha conminado a modificar sus prácticas alimentarias. [Hernández A, Velasco CM, Oviedo MP, Mantilla BP, Flórez NZ. Prácticas de alimentación de la población indígena del departamento de Chocó. Ustasalud 2014; 13(2):106 - 111]

Palabras clave: Seguridad alimentaria, Comunidades indígenas, Alimentación.

\section{FEEDING PRACTICES BY THE INDIGENOUS POPULATION OF DEPARTMENT OF CHOCÓ}

\section{ABSTRACT}

Objective: to identify conservation practices, handling, preparation and consumption of food of indigenous communities in the Department of Chocó

Methods: a descriptive qualitative study exploratory. We conducted a literature review part of the food security policy of the country and other inquiries with indigenous communities regarding their perception towards the practices addressed. We realize talk session with 185 people representing ethnic and territorial authorities, indigenous leaders in health, men, women, indigenous children.

Results: communities have shortcomings in terms of availability, access, consumption, utilization and quality of foods which are as critical to household food security of indigenous communities in the department.

Conclusions: the situation facing indigenous communities of Chocó is food insecurity also having little access to clean water, basic sanitation, among others, what has been ordered to change their dietary practices.

Key words: Food security, Indigenous communities, Feeding.

Recibido para publicación: 23 de octubre de 2014. Aceptado para publicación: 4 de diciembre de 2014 


\section{INTRODUCCIÓN}

La seguridad alimentaria y nutricional surge del reconocimiento del derecho de toda persona a no padecer hambre. Es la disponibilidad suficiente y estable de alimentos, el acceso a ellos y su consumo oportuno y permanente en cantidad, calidad e inocuidad, en condiciones que permitan su adecuada utilización biológica, para llevar una vida saludable y activa de todas las personas ${ }^{1}$.

En el ámbito mundial se han llevado a cabo diferentes espacios de encuentro ${ }^{2-4}$ entre naciones, organismos internacionales y sociedad civil, en torno a la temática, es así como, de acuerdo con la legislación nacional vigente ${ }^{5}$, se considera que ni en Colombia, ni en ningún país del mundo debería existir hambre, el derecho a la alimentación y la garantía del cumplimiento de este de manera prioritaria, ha venido reconceptualizándose como derecho a la seguridad alimentaria y nutricional ${ }^{6}$. Sin embargo, la carencia de alimentos para niñas, niños y población en general parecen ser el pan de cada día, en especial en algunas regiones del país como es el departamento de Chocó.

Según la definición planteada por la Organización de Naciones Unidas para la Agricultura y la Alimentación (FAO): Existe seguridad alimentaria cuando todas las personas tienen, en todo momento, acceso físico y económico a suficientes alimentos inocuos y nutritivos para satisfacer sus necesidades alimenticias y poder llevar así una vida activa y sana ${ }^{2}$. Este es un aspecto tan influyente en la calidad de vida y el desarrollo de un país que está directamente relacionado con tres de los Objetivos de Desarrollo del Milenio (ODM) y constituye un tema prioritario en la agenda de políticas públicas ${ }^{7}$. Así mismo, cuando se estudia la situación alimentaria y nutricional de un grupo, en este caso las poblaciones indígenas del departamento de Chocó, es necesario considerar aspectos como: el contexto social, el económico, el político, el histórico, el geográfico y el cultural ${ }^{8}$.

En Colombia se reconocen 102 pueblos indígenas, los cuales representan el 3\% del total de la población del país ${ }^{9}$, encontrándose en el departamento de Chocó las comunidades Embera Katío, Dóbida, Chamí y Wounaan. El presente artículo describe los resultados obtenidos a partir de una intervención realizada en dichas comunidades, con el objetivo de identificar las prácticas de conservación, manipulación, preparación y consumo de los alimentos de las comunidades indígenas.

\section{MATERIALES Y MÉTODOS}

Se realizó un estudio cualitativo descriptivo de tipo exploratorio ${ }^{10}$, para lo cual se hizo, por una parte, la revisión documental de la política de seguridad alimentaria a través de búsqueda bibliográfica y, por otra, las indagaciones con los participantes relacionadas con su percepción de las prácticas abordadas en el presente estudio, que se realizaron a través de momentos de conversación, siguiendo la propuesta metodológica de Taylor y Bogdan ${ }^{11}$.

Se llevaron a cabo diversos momentos de conversación, llamados "Encuentros de saberes", divididos en seis grupos diferentes con hombres y mujeres líderes en salud (jaibanas, tongueros, yerbateros, docentes, parteras) y familias de Chocó, los cuales se realizaron en dos espacios, así:

- Tres (3) grupos distribuidos así: 10 representantes de autoridades étnico-territoriales, 30 hombres y 45 mujeres líderes de las comunidades indígenas (embera katío, dóbida, chamí y waunaán).

- Encuentro con familias indígenas distribuidas en tres (3) grupos: 20 hombres, 20 mujeres, y 60 niñas y niños.

El número de asistentes a los encuentros de saberes obedece a la convocatoria realizada por los líderes en salud y a la masiva participación de las comunidades ante dicha convocatoria, para lo cual los grupos se subdividieron en un número de 10 a 15 personas para facilitar los espacios de conversación.

Se utilizaron diversas técnicas de recolección de la información adaptadas a las características propias de las comunidades indígenas, teniendo en cuenta que tienen una lengua propia, y los diferentes rangos de edades, por tanto se recurrió a presentación de imágenes (fotografías reales y animadas), historias (asociadas a la dinámica y realidad de las comunidades), ejercicios vivenciales, mapas comunitarios, lluvia de ideas, juegos, narración oral (historias, mitos, leyendas y juegos propios del contexto), las cuales permitieron establecer comunicación y profundizar frente a aquellos elementos relacionados con su seguridad alimentaria y nutricional.

A su vez, se realizó una revisión documental de la política de seguridad alimentaria del país, contrastando sus postulados con las categorías emergentes de los discursos y prácticas de las comunidades.

Los encuentros se llevaron a cabo en el periodo comprendido entre diciembre de 2010 a mayo de 2011 , las personas fueron contactadas a través de convocatoria directa realizada por los líderes en salud, quienes citaron a familias, mujeres y hombres de las diferentes comunidades. A las personas asistentes se les explicó los objetivos de los encuentros y los resultados esperados. Se les solicitó su consentimiento informado de manera verbal para la realización de los diferentes encuentros de saberes. Todos 
los encuentros fueron grabados en medio magnético, para posteriormente realizar el análisis de texto.

\section{Consideraciones éticas}

En todo momento de la investigación se realizó un manejo ético, anónimo y confidencial de la información, respetando las normas de investigación en sujetos humanos, estipuladas por la declaración de Helsinki II y la Resolución 008430 de 1993 del Ministerio de Salud de Colombia ${ }^{12}$ por la cual se establece las normas científicas para la investigación en salud. Para el caso de la participación de las niñas y los niños se solicitó permiso y aprobación verbal a sus familias.

\section{RESULTADOS}

Las políticas públicas están dirigidas a la creación de soluciones específicas, de cómo manejar asuntos públicos o situaciones conjuntas que se convierten en condiciones socialmente problemáticas $\mathrm{y}$, por tanto, afectan negativamente al bienestar de la población, es así como la política de Seguridad Alimentaria se constituye como la vía para dar respuesta a sus necesidades en salud y garantizar derechos fundamentales, mejorando las condiciones que afectan de manera adversa el bienestar de las comunidades.

La política plantea unos ejes para garantizar el ejercicio de los derechos y deberes que requiere la acción del Estado, la sociedad civil y la familia, y define las condiciones necesarias y suficientes para lograr la seguridad alimentaria y nutricional ${ }^{1}$, dichos ejes son analizados en el presente estudio en el contexto de las comunidades indígenas del departamento de Chocó.

Teniendo en cuenta el abordaje realizado, se presentan a continuación los resultados de manera integrada, mencionando los elementos relatados por las comunidades indígenas en relación con sus prácticas de alimentación y el panorama dado por ejes desde la política de seguridad alimentaria del país.

El fin último de la seguridad alimentaria es que todas las personas tengan una alimentación suficiente, oportuna y adecuada, es así como en Colombia se aprueba, como una política de Estado, la política de seguridad alimentaria y nutricional PSAN ${ }^{1}$, la cual contempla los siguientes ejes, que a continuación se presentan de manera integrada entre el análisis documental y los hallazgos reportados: En la figura uno se observan los elementos que condicionan la seguridad alimentaria de las comunidades indígenas, relacionadas con los ejes de la PSAN.

1. Disponibilidad: entendida esta como la cantidad de alimentos con que se cuenta. Está relacionada con el suministro suficiente de estos en rela- ción con los requerimientos de la población. En cuanto a este eje, se identifica desde la perspectiva de las comunidades, que tienen dificultades marcadas en la disponibilidad de alimentos, pues la tierra tiene usos como cultivos ilícitos, lo cual está afectando la fertilidad, por ende, los cultivos, la caza y la pesca ha disminuido, así como las variedades. Todo esto por las condiciones sociales, políticas, económicas y de conflicto que vive el departamento, que por supuesto afecta directamente a la población.

Lo anterior se ratifica en lo mencionado por la comunidad referente a los cultivos, "los grupos al margen de la ley los están obligando a sembrar coca, que ya el resto de cultivos no, nada, se los están prohibiendo, que en adelante era todos a sembrar coca, entonces esas personas corren el riesgo, tienen dos tipos de riesgos allí, tienen dos problemas encima: el problema alimentario y el problema de orden público".

En cuanto a la pesca mencionan: "En nuestro rio está acabado el pescado, ya no hay que pescar. La violencia nos ha desplazado, cuando uno vuelve ya no hay nada, ellos han acabado con todo".

2. Acceso: hace referencia a los alimentos que pueden obtener o comprar las familias y comunidades, en este sentido es necesario considerar varios factores: según los relatos de las comunidades se identificaron: dificultades para el acceso a la educación, lo que afecta directamente las condiciones laborales y los ingresos; las ocupaciones en la zona, se caracterizan por la agricultura, minería y pesca, las cuales se han visto afectadas por las condiciones ambientales, el conflicto armado, la minería y los cultivos ilícitos, entre otros, en este sentido los ingresos también se ven condicionados; adicionalmente se encuentran las barreras geográficas, los traslados son largos y costosos, ya que implican hacer uso de las vías fluviales y terrestres, "el sitio donde se compra está a nueve horas de camino, y algunos productos como el plátano ya no se está dando, por tanto hay que comprarlo".

Así mismo se encuentra la barrera de los costos de los alimentos, y de la escasez de dinero para acceder a los mismos, lo cual ha disminuido la alimentación por falta de acceso, "ahora no estamos sembrando plátano, pues de la tierra ya no sale, el Dios ya no da, ahora lo compramos cuando hay plata, pero cuando no hay para la comida bien jodido".

3. Consumo: se refiere a los alimentos que comen las personas y está relacionado con la selección de estos, las creencias, las actitudes y las prácticas; las comunidades realizan tres tiempos de 


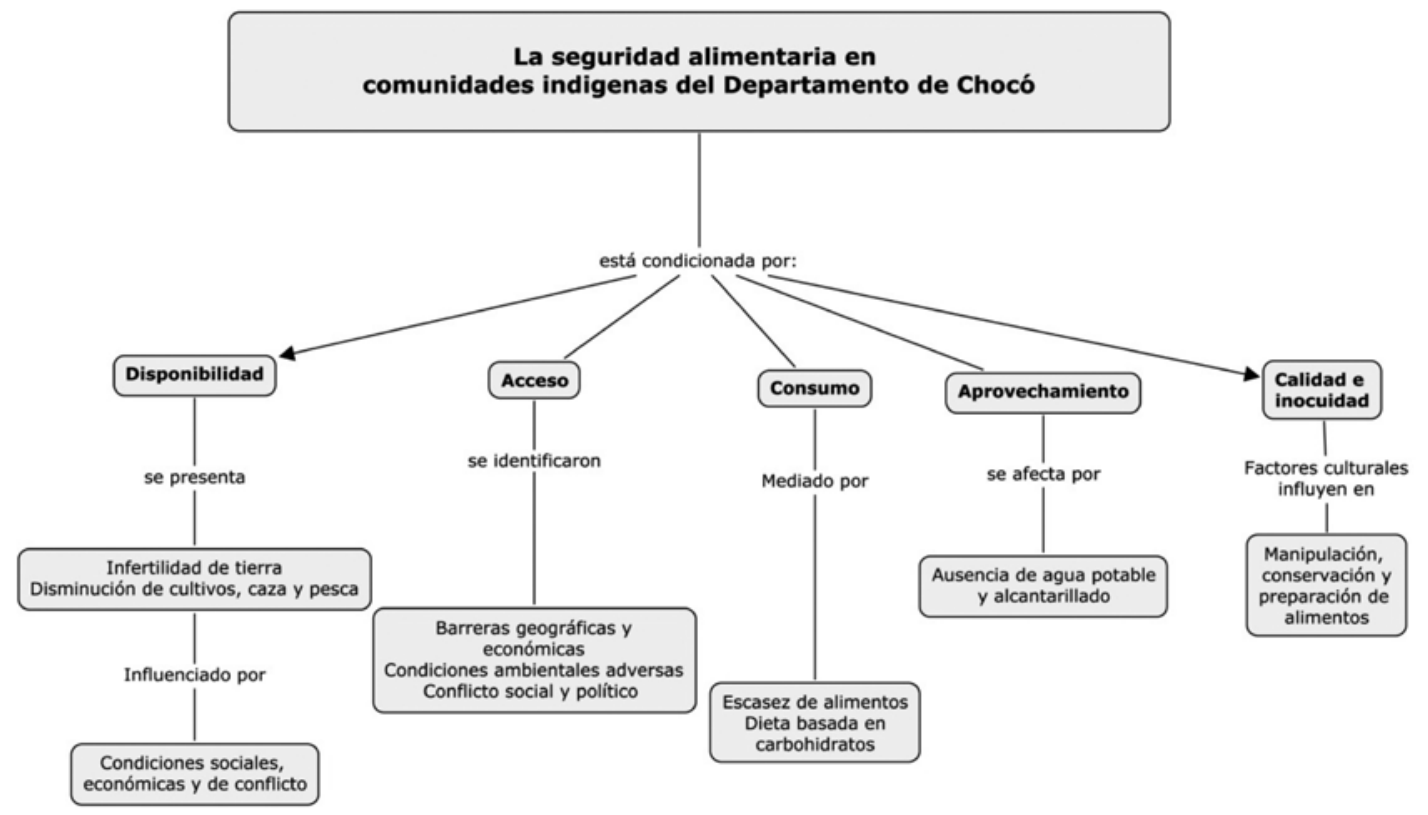

Figura 1. Elementos que condicionan la seguridad alimentaria de las comunidades indígenas del Departamento de Chocó. Fuente: Elaboración propia.

comida: la alimentación se basa en plátano, que acompañan con arroz y chicha y por la escasez, ocasionalmente ingieren carne o pescado. En este sentido, y dado que se encuentran problemas por la siembra y cultivo de plátano, se han visto obligados a modificar su dieta alimentaria. No toman leche de vaca, pues este tipo de ganadería no es común en la zona, la leche la obtienen de una palma llamada "mil pesos". En relación con la ingesta de verduras expresan no incluirlas en su dieta diaria. Las frutas las consumen recién bajadas del árbol, encontrándose que cultivan chontaduro, borojó, papaya, limón, maduro, banano, guayaba y piña, además cuentan con azoteas y huertas caseras, donde siembran aguacate, marañón y caimito.

4. Aprovechamiento o utilización biológica: para el presente caso se hará referencia únicamente a la disponibilidad, la calidad y el acceso a agua potable y saneamiento básico. La cobertura de acueducto en el departamento es del $22,5 \%{ }^{13}$. La población indígena utiliza las aguas lluvias y el agua de ríos y quebradas para suplir sus necesidades, "sacamos el agua del estanque, tenemos tanques, tenemos bastante agua de la lluvia, cuando no llueve, buscamos quebraditas, la cargamos a la casa cocinamos, bebemos y para lavar platos también". La cobertura de alcantarillado es del $15,9 \%$, las personas utilizan las letrinas, el campo abierto o ríos y quebradas como alcantarillado, "la gente hace chichi y popo en el río".
5. Calidad e inocuidad: se refiere al conjunto de características de los alimentos que garantizan que sean aptos para el consumo humano, incluye la manipulación, conservación y preparación de los alimentos. Para la manipulación de los alimentos, "algunas mujeres se lavan las manos con agua antes de cocinar, pero la mayoría no lo hacen".

En cuanto a la conservación "si tienen huevos se guardan en un canasto pequeño, si se pesca se consume ahí mismo, si se coge bastante se le echa buena salecita y se guarda, la carne la ahumamos, se sala y no le pasa nada, el plátano lo ponemos en el piso, el arroz lo guardamos en bolsas que metemos en baldes".

La preparación de los alimentos es preponderantemente tarea de las mujeres, "la mujer cocina y el hombre es el que trae la comida del monte". El agua utilizada para preparar los alimentos y para el consumo es extraída del río o de la recolección de aguas lluvias.

\section{DISCUSIÓN}

Identificar las prácticas de alimentación de las comunidades indígenas del departamento de Choco que participaron en el estudio, permitió aportar evidencia y reconocer las diversas limitaciones y dificultades que presentan dichas comunidades para gozar de seguridad alimentaria.

Al comparar la información obtenida en los diversos momentos de conversación con las comunidades y con fuentes de información o estudios previos, se 
identifica cómo los discursos de los entrevistados son coincidentes con los registros oficiales, lo cual ratifica las precarias condiciones que viven las comunidades del departamento de Chocó. Los resultados obtenidos permiten identificar una serie de condiciones que obstaculizan la seguridad alimentaria de las comunidades indígenas, dados en: carencia de alimentos para el consumo y barreras sociales, geográficas y económicas para el acceso a alimentos, las cuales han sido reportadas en otros estudios con diferentes enfoques metodológicos.

En este sentido, al revisar los datos de la Gobernación del departamento ${ }^{14}$, en cuanto al consumo de alimentos y percepción de seguridad alimentaria en el hogar, se evidencia que en relación con el consumo de alimentos de los diferentes grupos de población: el $17 \%$ de la población no había consumido cárnicos el día anterior a la encuesta, el 44\% no había consumido lácteos, el $31,2 \%$, frutas; y el $39,7 \%$, verduras. Esta compleja situación de seguridad alimentaria, ha sido reportada en otro estudio, realizado con una muestra de población indígena del departamento perteneciente a los pueblos Embera Dobida, Embera Katío, Embera Chamí, Tule y Wounaan, en el cual se mostró que el 99,9\% de los hogares se encuentra en inseguridad alimentaria y el $94,9 \%$ en inseguridad alimentaria severa ${ }^{15}$.

En el departamento de Choco, la tasa de desnutrición crónica para los niños menores de 5 años es de $42,4 \%{ }^{9}$, donde el grupo más afectado fue la comunidad Wounaan ${ }^{8}$. Así mismo, se han reportado situaciones complejas en relación con el estado nutricional de comunidades indígenas, en los pueblos Tule, en los que se encontró que un $80 \%$ los niños de 0 a 11 años estaban en riesgo de desnutrición crónica, en los resguardos Embera-Katío, Tierralta, Córdoba, la prevalencia de desnutrición crónica infantil es del 63,6\% ${ }^{9}$.

Así mismo, al revisar la literatura existente, se identifican condiciones similares en cuanto a ingesta de alimentos por parte de las comunidades indígenas en México, con los grupos de indígenas otomíes, que presentan condiciones económicas difíciles y de productividad de la tierra, donde el consumo de alimentos es bajo, no consumen casi carne, leche, frutas ni verduras ${ }^{16}$. De la misma manera un estudio realizado en Perú demuestra la deficiencia en nutrientes en la alimentación de la comunidad indígena, en la que la dieta se basa en yuca y plátanos, y pocos alimentos de origen animal ${ }^{17}$.

Al igual que lo encontrado en la presente investigación, se identifican otros estudios donde los pueblos indígenas han modificado sus tradiciones de la dedicación a la caza, pesca y recolección por dificulta- des con la sobreexplotación de su entorno, el agotamiento de los recursos naturales, contaminación de las aguas de los ríos por las zonas urbanas y el uso indiscriminado de elementos nocivos para la pesca, y por ende ha disminuido la fuente de proteínas que aportaban la caza y pesca ${ }^{8,18}$.

El conflicto social vivido en el departamento de Chocó es comparable con otras zonas de comunidades indígenas, dicha situación ha sido reportada como un factor que las afecta, dado que la irrupción de los violentos en su existencia propicia el desplazamiento forzado y con ello, la violación del derecho a la seguridad alimentaria ${ }^{19}$, tal y como lo muestran estudios, tanto nacionales como en comunidades de México ${ }^{20}$, donde se identifica cómo la migración y un ingreso económico insuficiente disminuye la posibilidad de contar con una dieta variada y de calidad.

Al igual que lo evidenciado en el presente estudio con las comunidades indígenas del Chocó, un estudio realizado sobre el estado nutricional de indígenas emberas $^{21}$ encontró que la inseguridad alimentaria surge de un conjunto de causalidades, entre las que destacan, los hábitos alimentarios y las dificultades de acceso a los alimentos, los cuales, afectan el estado nutricional de la población; de la misma manera un estudio con la comunidad indígena tules ${ }^{22}$ demuestra las deficiencias alimentarias y la relación directa con la desnutrición de esta población, situaciones agravadas por la carencia de tierras fértiles y las dificultades que tienen con la caza y pesca.

\section{Conclusiones}

El departamento de Chocó es una zona con gran riqueza en fauna, flora; paradójicamente la situación que viven las comunidades indígenas es de inseguridad alimentaria, tienen dificultades de acceso al agua potable, saneamiento básico, pobreza, situación que se ha aumentado por la minería a gran escala, por los cultivos ilícitos, por el conflicto armado, que ha conminado a sus habitantes a modificar sus hábitos alimentarios, por varias razones que afectan la provisión y disponibilidad de alimentos.

Los ríos se han contaminado, por ende los peces han disminuido, la entrada de maquinarias por la minería en las tierras han ocasionado que los animales de caza hayan migrado, los cultivos ilícitos han ocasionado que la tierra se haya ido esterilizando, en una palabra, poco a poco se ha menguado la fuente de alimentos, adicionalmente, la relación de la población indígena con las tradiciones occidentales de alimentación han generado que cambien sus hábitos, situaciones que están provocando que las comunidades padezcan desnutrición, que afecta en mayor medida a los niños y a las gestantes. 


\section{Agradecimientos}

A las comunidades indígenas (Embera Katío, Dóbida, Chamí y Wounaan), autoridades étnico-territoriales. A los doctores Lucila Niño Bautista, Gloria Marconi y Yan Carlos Cataño Montoya por la revisión y aportes realizados al artículo.

\section{Nota aclaratoria}

Por motivos de seguridad de las personas participantes en el proceso de recolección de información, se omiten los nombres, perfiles y roles desempeñados por cada una en las citas referenciadas en el texto.

\section{Conflicto de intereses}

Las autoras manifiestan no tener conflicto de intereses.

\section{BIBLIOGRAFÍA}

1. Documento Conpes Social 113, Consejo Nacional de Política Económica Social, República de Colombia, Política Nacional de Seguridad Alimentaria y Nutricional (PSAN), (2007).

2. ONU (Organización de las Naciones Unidas). [Internet] Roma: Cumbre Mundial sobre la Alimentación 1996; [citado 2012 Nov 25]. Disponible en: http://www.cinu.org. $\mathrm{mx} /$ temas/desarrollo/dessocial/alimentos/dec_plan_aliment1996.htm\#d\%20ec;\%201996.

3. ONU (Organización de las Naciones Unidas). [Internet] La Asamblea del Milenio de las Naciones Unidas, 2000; [Citado 2012 agosto 2]. Disponible en: http://www.un.org/ spanish/milenio/

4. Naciones Unidas [Internet]. Podemos erradicar la pobreza; [Citado 2012 sept 20]. Disponible en: http://www. un.org/es/millenniumgoals/.

5. El Pueblo de Colombia, Constitución Política de Colombia. Artículos 44, 64, 65, 66 (1991).

6. Del Castillo S, Chacón O, Giraldo M, Botero J. Observatorio de seguridad alimentaria y nutricional. Universidad Nacional de Colombia, Secretaría Distrital de Salud. Convenio 0644-2007.

7. Ramos E, Salazar GI, Berrún LN, Zambrano A. Reflexiones sobre derecho acceso y disponibilidad de alimentos. Revista de Salud Pública y Nutrición. 2007;8(4). Disponible en: http://www.respyn.uanl.mx/viii/4/ensayos/accesodisponibilidad-bba.htm

8. Tobar L, Chinchilla M. Aspectos nutricionales y alimentarios de las comunidades indígenas colombianas. Instituto colombiano de cultura hispánica, geografía humana de Colombia variación biológica y cultural en Colombia (tomo I). Facultad de Ciencias. Pontificia Universidad Javeriana. Disponible en: http://www.banrepcultural.org/ blaavirtual/geografia/geofraf1/aspectos.htm

9. Comunidad Andina. Secretaría General. Décimo séptima reunión del grupo ad hoc de seguridad alimentaria para poblaciones indígenas en la comunidad andina videoconferencia. Información sobre seguridad alimentaria y nutricional de la población indígena en los países de la comunidad andina. [Internet] $2010 \mathrm{feb} 26$. Disponible en: http://www.comunidadandina.org/upload/20114131833 15informacsapidt.doc
10. Ulin $\mathrm{R}$, Robinson $\mathrm{E}$, Tolley E. Investigación aplicada en salud pública: métodos cualitativos. Rev. Inst. Med. trop. S. paulo [Internet] . 2007 abr [citado 2012 sep 12]; 49(2):86-86. Disponible en: http://www.scielo.br/scielo.php? script $=$ sci_arttext \&pid $=$ S003646652007000200015\&lng=en.http://dx.doi.org/10.1590/ S0036-46652007000200015

11. Taylor S.J, Bogdan R. Introducción a los métodos cualitativos de investigación. Paidós Barcelona, 1987. p 152-160.

12. República de Colombia, Ministerio de Salud. Resolución 008430 de 1993.

13. Gobernación del Chocó. Plan de Desarrollo del Departamento del Chocó 2008-2011: "Un giro para salvar al Chocó". 2008.

14. Chocó. Plan Departamental de Seguridad Alimentaria y Nutricional del Chocó. (BITUTE). Medellín: Centro Imagen Universidad Pontificia Bolivariana. 2007.

15. Fondo Español para el Logro de los Objetivos del Milenio (FODM). Las comunidades indígenas y afrocolombianas promueven su seguridad alimentaria y nutricional, Ventana de seguridad alimentaria y nutrición. (En línea). Mayo 2009 (citado 2013 mayo 08). Disponible en: http://www.pnud.org.co/img_uplo ad/36353463616361636163616361636163/72828 MDGF_SEGURIDAD_ALIMENTARIA_CHOCO.pdf

16. Anderson RK, Calvo J, Serrano G, Payne GC. Estudio del estado de nutrición y los hábitos alimentarios de comunidades otomíes en el Valle del Mezquital de México. Salud pública México 2009;51:657-674.

17. Huamán-Espino L \& Valladares C. Estado nutricional y características del consumo alimentario de la población Aguaruna. Amazonas, Perú 2004. Rev Perú Med Exp Salud Pública. 2006;23(1). 12-21

18. Huamán-Espino E. Más allá de la estadística alimentaria y nutricional. Percepciones de un Aguaruna con relación a la situación alimentaria de su pueblo. Rev Perú Med Exp Salud Pública. 2006;23(1):73-6.

19. Santa J, Gálves A. El plátano vacío: conflicto armado y hábitos alimentarios en el pueblo Embera de Frontino Colombia. Zainak. 2008;30:195-207.

20. Reyes I, Beutelspacher N, Estrada E, Mundo V. Alimentación y suficiencia energética en indígenas migrantes de los Altos de Chiapas, México. ALAN 2007 Jun;57(2):155162.

21. Rosique J, Restrepo MT, Manjarrés LM, Gálvez A, Santa J. Estado nutricional y hábitos alimentarios en indígenas Embera de Colombia. Rev. Chil. Nutr. [Internet]. 2010 Sep (citado 2012 Julio 03). Disponible en: http://www. scielo.cl/scielo.php?script $=$ sci arttext\&pid $=$ S0717$75182010000300002 \& \operatorname{lng}=$ es

22. Carmona J, Correa A, Alcaraz G. Población, alimentación y estado nutricional entre los tules (kunas) del resguardo Caimán Nuevo (Turbo y Necoclí; Antioquia, Colombia), 2003-2004. IATREIA. 2005 sep;18(3):259-278.

\section{Correo electrónico de los autores:}

Andrea Hernández Quirama: ahernanq@uis.edu.co Claudia Milena Velasco Rangel: mile0833@hotmail.com Blanca Patricia Mantilla Uribe: bpmantil@uis.edu.co Nieves Zoraida Lórez García: nizoflo@yahoo.com 